Message passing for the coloring problem: Gallager meets Alon and Kahale 


\title{
On expected number of maximal points in polytopes
}

\author{
Yu. Baryshnikov ${ }^{1 / \hbar}$ \\ ${ }^{1}$ Mathematical Sciences, Bell Laboratories, \\ Murray Hill, NJ, USA \\ revised January 19, 2008,
}

We answer an old question: what are possible growth rates of the expected number of vector-maximal points in a uniform sample from a polytope.

Keywords: -

\section{1 introduction}

\section{1 setup}

We consider the following setup

- Fix a closed convex polyhedral cone $K$ with nonempty interior in $d$-dimensional Euclidean space $V=\mathbb{R}^{d}$

- The cone $K$ defines a partial ordering on $V:$ for $x, y \in V$,

$$
x>_{K} y \quad \text { iff } \quad x-y \in K
$$

(we say that $x$ dominates $y$ or that $y$ is dominated by $x$ ).

- a point $x \in X$ is said to be $K$-maximal, or simply maximal if there are no further point $x^{\prime} \in X$ dominating $x$.

(We will be using notation

$$
\max _{K}(X) \text {. }
$$

for the set of maximal points in a set $X \subset V$.)

- Assume further that a convex (compact) polyhedron $P$ is given, and that

\footnotetext{
$\dagger$ Suppoorted in part by ARO

1365-8050 @ 2007 Discrete Mathematics and Theoretical Computer Science (DMTCS), Nancy, France
} 
- $X$ is a uniform size $n$ sample from $P$.

The following question arises in various contexts:

Question 1 Given $P$ and $K$, find the expected size

$$
M_{n}=\mathbb{E}\left|\max _{K}(X)\right|
$$

of $K$-maximal elements in $X$, as a function of the sample size $n=|X|$.

I will not try to survey here all situations where computing $M_{n}$ can be useful, just mention some keywords - multicriterial optimization, geometric algorithms, convex hulls - and refer the reader to [6, 8 , [4, 5, ?]

\subsubsection{Convention}

We will use $f \approx g$ as a synonym for

$$
\frac{f}{g} \rightarrow c, 0<c<\infty .
$$

\section{2 what was known so far}

The Question 1 was addressed by many authors having different applications in mind; consequently, they arrived at partial answers. Two of the possible setting studied most occupy in some sense opposite corners of the space of all problems:

- If $P$ is the unit square, and $K$ is the positive quarter plane (in $d=2$ ), the number of maximal point is the same as the number of records in an iid sample, i.e. harmonic number

$$
H_{n}=\sum_{i=1}^{n} \frac{1}{i} .
$$

More generally, in higher dimensions, if $P$ is the unit cube, and $K$ is the positive orthant ("Pareto cone"), the problem still is essentially combinatorial, and the expected number of maximal elements is the incomplete polyzeta: thus, in dimension $d$, the expected number of maximal elements is

$$
M_{n}=\frac{1}{n} \sum_{1 \leq i_{1} \leq . \leq \cdots \leq i_{d-1} \leq n} \frac{1}{i_{1} i_{2} \cdots i_{d-1}} .
$$

(this result was first established, it seems, in [2], and reproduced by many authors).

- If the polyhedron $P$ is in general position with respect to the cone $K$ (meaning: all flats spanned by facets of $K$ and $P$ intersect transversally), then

$$
M_{n} \approx n^{f / d}
$$

where $f$ is the dimension of $\max _{K}(P)[3]$.

The situations described above are in some sense the most degenerate and most generic ones, respectively. In the former case, all faces of $P$ are parallel to some faces of $K$. Generically, a small perturbation would lead to a $K, P$ being in general position. Intermediate situations are relevant, however: for example, if $P$ is given as a set of solution of a system of linear inequalities $P=\{A x \leq b\}$, the sparsity of the matrix $A$ would lead to a problem intermediate between the those above. 


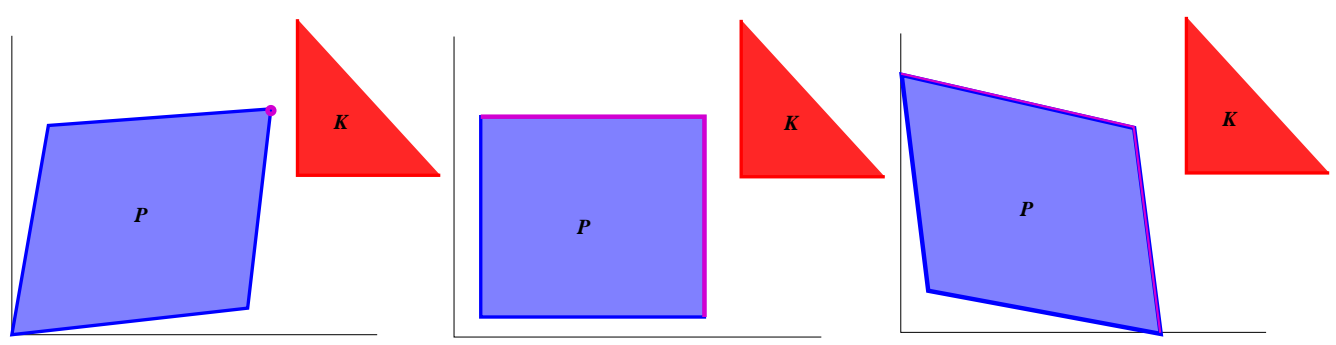

Figure 1: Generic deformations of the polyhedron $P$ : in a smooth 1-dimensional family, typical representative has polynomial growth ( $M_{n} \approx n^{0}$ on the left; $M_{n} \approx n^{1 / 2}$ on the right), and has logarithmic terms for exceptional values of parameter $\left(M_{n} \approx \log n\right.$ for the middle display).

\section{3 gap problem}

It has been noticed [8] that there is a certain gap in the possible asymptotic behaviors of $M_{n}$ as a function of $n$, at least in dimension 2 : if the expected number grows faster than $\log (n)$, then it is asymptotically $\Omega(\sqrt{n})$. This, clearly, motivates the problem:

Question 2 What are the possible asymptotics of $M_{n}$, at least for convex polyhedral $K, P$ (and uniform sample from $P$ )?

In other words, what else, beyond observed so far behaviors $M_{n} \approx n^{f / d} ; M_{n} \approx \log ^{d-1}(n)$ can happen within our polyhedral setup?

In this work we answer both Questions, 1 and 2 .

\subsection{1 acknowledgment}

I was told about the gap problem by Mordecai Golin during AofA'06; many thanks!

\section{2 main result}

Consider the set of points

$$
\Delta=\left\{(m, c) \in \mathbb{Z}^{2}, 0 \leq c \leq m \leq d\right\} .
$$

We will call a pair $(r, \mu), r>0, \mu \in \mathbb{N}$ admissible, if the intersection of the ray

$$
c=r m, m \geq 0
$$

with the set $\Delta$ contains at least at least $m u$ points (beyond the origin). In particular, in an admissible pair $(r, \mu), r$ is rational.

Theorem 1 The expected number of maximal elements $M_{n}=\mathbb{E}\left(\max _{K}(X)\right)$ with respect to a convex closed polyhedral cone $K$, where $X$ is the uniform sample from polyhedral $P \subset V$ of size $n$, satisfies

$$
M_{n} \approx n^{1-r} \log ^{\mu-1}(n),
$$

for some admissible pair $(r, \mu)$. For any admissible $(r, \mu)$, there exists a pair $(P, K)$ satisfying $(1)$. 

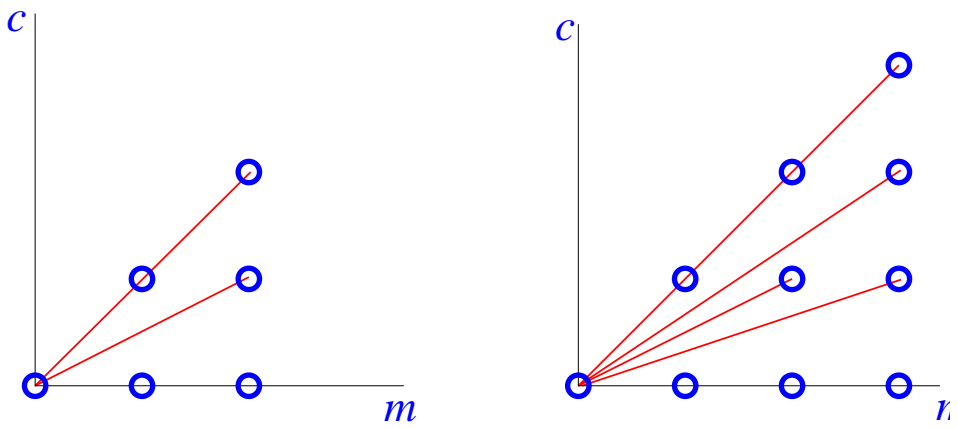

Figure 2: Asymptotics of $M_{n}$ in dimensions 2,3.

\section{1 examples}

1. For $d=2$, the Figure 2.1 shows all possible alternatives: the point $(m, c)=(1,1)$ corresponds to $M_{n} \approx n^{0}$ (compare Fig. 1.2. left); the point $(2,2)$ correspond to $M_{n} \approx \log n$ (Fig. 1.2 . middle) and the point $(2,1)$ corresponds to $M_{n} \approx n^{1 / 2}$. In particular, one can see the "gap".

2. For $d=3$, asymptotic growth rates for $M_{n}$ are

- $M_{n} \approx n^{1-r}, r=1 / 3,1 / 2,2 / 3$;

- $M_{n} \approx \log ^{\nu}(n), \nu=0,1,2$.

3. For illustrative purposes, some examples in dimension 8: The three segments illustrate the following

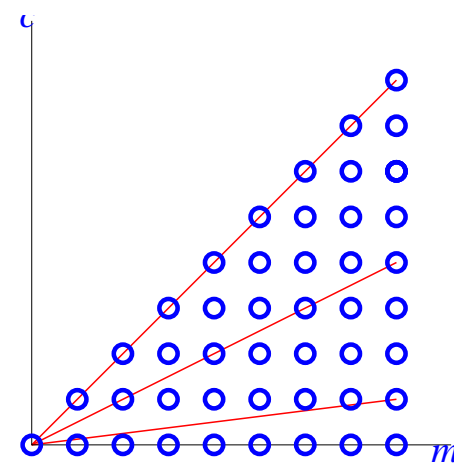

Figure 3: Asymptotics of $M_{n}$ in dimension 8 .

asymptotics (bottom-to-top):

- $M_{n} \approx n^{7 / 8}$

- $M_{n} \approx n^{3 / 4} \log ^{3}(n)$; 
- $\log ^{7}(n)$

Remark: In generic situation (when all faces of $P$ are transversal to all faces of $K$ ), all multiplicities $\mu$ are equal to 0 .

\section{2 specific polytopes and cones}

The general result of the previous section depends, of course, on the results describing the asymptotics of $M_{n}$ for specific instances of $(P, K)$. To describe those, we will need to define flags and their spectra.

Consider the (ranked) face poset of the polytope $P$ : its elements are faces of $P$ of all dimensions ordered by inclusion (thus the maximal face is of dimension $d$, the polytope $P$ itself, then the facets of $P$ of dimension $d-1$ and so on. We will denote the set of faces of dimension $l$ as $\mathcal{P}_{l}$.

Flags are the chains of adjoining proper faces of $P$ :

$$
\mathcal{F}=\left(f_{1}, f_{2}, \ldots, f_{l}\right): f_{1} \subset f_{2} \subset \ldots \subset f_{l}, f_{i} \in \mathcal{P}_{d_{i}} ; d_{l}<d .
$$

A flag if full if it has length $d$, i.e. it includes facets of all dimensions between 0 and $d-1$.

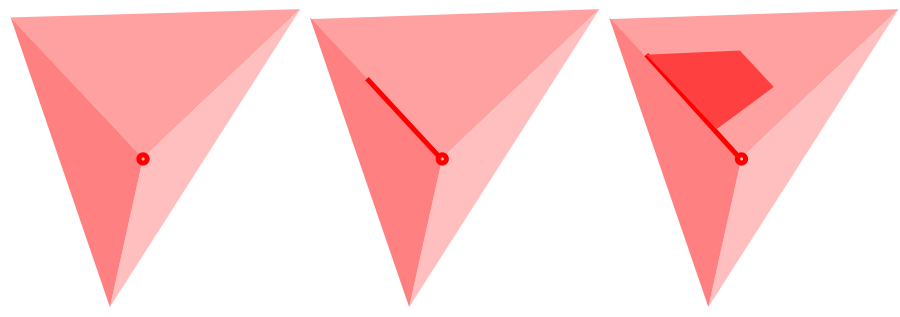

Figure 4: A flag.

We associate to each face $f$ of $P$ its deficiency $\delta(f)$ : it is just the dimension of the intersection

$$
(K+x) \cap P
$$

for a (relative) interior point $x \in f$. One can easily check that the deficiency is well-defined, i.e. does not depend on particular point in the relative interior of a face.

Deficiencies in a flag do not decrease:

$$
\delta\left(f_{1}\right) \leq \delta\left(f_{2}\right), f_{1} \subset f_{2} .
$$

Definition: The spectrum of a flag $F=\left(f_{1} \subset f_{2} \subset \ldots \subset f_{l}\right)$ is the multiset

$$
\sigma(\sigma)=\left\{\frac{c\left(f_{1}\right)}{m\left(f_{1}\right)}, \frac{c\left(f_{2}\right)}{m\left(f_{2}\right)}, \ldots, \frac{c\left(f_{l}\right)}{m\left(f_{l}\right)}\right\}
$$

(where elements are counted with multiplicities), where

$$
c\left(f_{i}\right)=\operatorname{codim}\left(f_{i}\right)=d-\operatorname{dim}\left(f_{i}\right)
$$



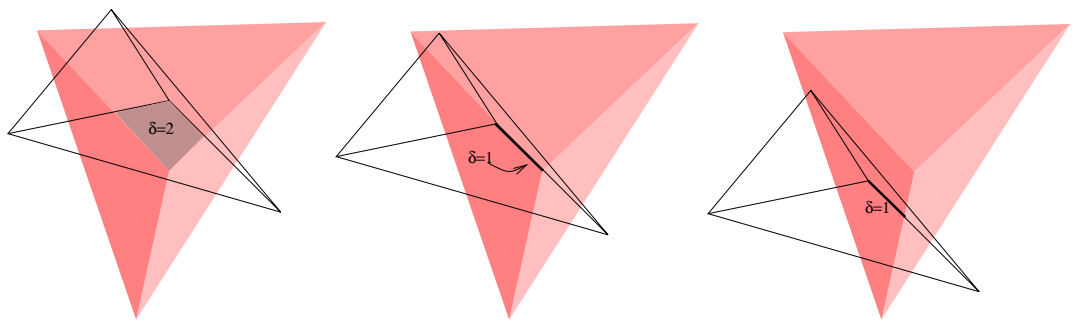

Figure 5: Deficiencies of some of the facets for polytope on Figure 2.2 the deficiencies of the facets in the flag on Figure 2.2 are equal to $(0,1,2)$, and its spectrum is $\left\{1^{3}\right\}$ (1 with multiplicity 3$)$.

is the codimension of a face, and

$$
m\left(f_{i}\right)=d-\delta\left(f_{i}\right)
$$

is its codeficiency.

Definition: For a flag $\mathcal{F}$ define its leading exponent to be

$$
r(\mathcal{F})=\min \{r: r \in \sigma(\mathcal{F})\},
$$

the smallest number in the flag spectrum. The multiplicity $\mu(\mathcal{F})$ is the multiplicity of $r(\mathcal{F})$ in $\sigma(\mathcal{F})$.

The spectra of flags are crucial for us due to our second main result,

Theorem 2 The asymptotics of $M_{n}$, as $n \rightarrow \infty$ is given by

$$
M_{n} \approx n^{1-r_{*}} \log ^{\mu-1}(n),
$$

where $r_{*}=\min _{\mathcal{F}} r(\mathcal{F})$ is the smallest of the leading exponents of flags, and $\mu$ is the largest multiplicity of $r_{*}$ among all the flags.

Remark: It is immediate that extending a flag only increases its spectra, and thus can lead only to faster growing terms in (2). Hence to analyze the leading asymptotics of $M_{n}$ it is enough to consider only full flags.

Remark: In fact, the entire asymptotic expansion of $M_{n}$ is governed by the spectra of flags. Essentially, for each pair $(c(\mathcal{F}), m(\mathcal{F}))$ such that $r=c / m$ occurs in the flag spectrum with multiplicity $\mu$ contributes to the asymptotic expansion of $M_{n}$ an asymptotic series

$$
\sum_{0 \leq l ; 0 \leq \nu<\mu} a_{l, \nu} n^{1-(c+l) / m} \log ^{\nu}(n) .
$$

We will not go into details, postponing a detailed exposition of this (and all the remaining) topics to a separate paper.

\section{3 techniques}

The techniques are a melange of Fubini theorem, an elementary version of resolution of singularities and some fairly standard results from the theory of generalized functions. We will not even attempt to present any details of the proofs (which, while not complicated, would require quite a bit of supporting machinery), but rather will sketch the major steps and outline main constructions. 


\subsection{Fubini}

We will denote by $\lambda$ the Lebesgue measure on $V$, and by $|P|=\lambda(P)$ the volume of the polytope $P$.

For a point $x \in P$, denote by

$$
u(x)=|P|^{-1} \lambda(K+x \cap P)
$$

the probability that a random point from $P$ dominates $x$.

Lemma 1 The function $u$ is piece-wise polynomial in $V$ : there exists a polyhedral subdivision of $P$ (spline subdivision) such that the restriction of $u$ to each of its polyhedra is polynomial.

We will use the following formula which is a more or less straightforward corollary of Fubini and a formula for $M_{n}$ implied by conditioning on the positions of a point in $X$ and finding the probability that this point is maximal:

Lemma 2

$$
M_{n}=n \int(1-t)^{n-1} d B(t)
$$

where

$$
B(t)=|P|^{-1} \lambda\{x \in P: u(x) \leq t\},
$$

is the probability that $M$ evaluated at a random point in $P$ is $\leq t$.

The advantage of using (3) is the decoupling of geometry: we can concentrate now on the properties of the function $B(t)$. Indeed, Karamata-type Tauberian theorems would translate the asymptotics of $B$ near zero into the the asymptotics of $M_{n}, n \rightarrow \infty$.

To analyze $B$ near 0 , we need to analyze $u$ near the points where $u$ vanishes. This can be done locally.

\section{2 resolution of singularities}

The domains where $u$ is polynomial can adjoin the faces of $P$ in rather intricate fashion. Hence a resolution of $P$ would be helpful. In particular, it would be convenient to arrive at the domain with controlled singularities, specifically, with normal intersections (where each face of codimension $k$ is adjoined to at most $k$ faces of codimension 1 ).

To do so we resolve the singularities of $P$ (i.e. its faces of codimension 2 and higher), in such a way that all facets (independently of their dimensions) would lift to faces of dimension $(d-1)$ in the resolved polytope, the flags of length 2 (pairs of incident facets) would lift to faces of dimension $(d-2)$ and so on, with full flags corresponding to the vertices in the resolution.

The easiest way to visualize such a resolution is as follows:

For each face $f^{l}$ of dimension $l<d$ of the polytope $P$ consider the $\epsilon^{l+1}$-tube $T(f)$ around this face (i.e. the set of all points in $P$ at the distance at most $\epsilon^{l+1}$ from the affine subspace (of dimension $l$ ) spanned by $f^{l}$. For small enough $\epsilon$ this tubes intersect transversally, and only if the corresponding faces adjoin.

Let $P_{\epsilon}$ be the complement to the union of these tubes,

$$
P_{\epsilon}=P-\cup_{l<d} \cup_{f^{l} \subset P} T(f) .
$$

It is immediate that the facets of $P_{\epsilon}$ are in one-to-one correspondence with flags of $P$. 

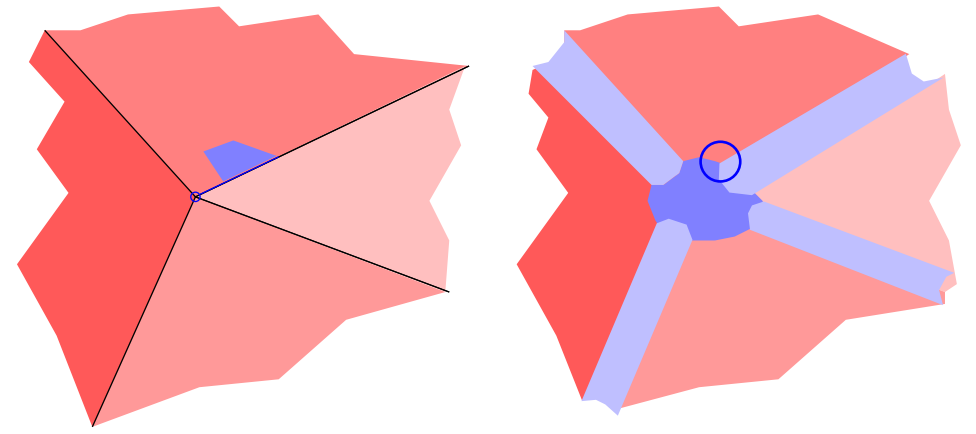

Figure 6: Resolution of vertices: non-simple vertex becomes (topologically) simple after the resolution. Vertices of the resolved polytope correspond to the flags of the original polytope $P$ : one such pair, flag $\leftrightarrow$ vertex, is shown.

Proposition 1 The (topological) polyhedron $P_{\epsilon}$ resolves $P$ : there exists a (natural) mapping $\pi: P_{\epsilon} \rightarrow P$ which is a diffeomorphism on the interior of $P_{\epsilon}$ taking each codimension 1 facet of $P_{\epsilon}$ to the corresponding facet of $P$. (More generally, the faces of $P_{\epsilon}$ of codimension $k$ correspond to flags in $P$ of length $k$.) Further, the polyhedron $P_{\epsilon}$ is a manifold with corners: locally, it is diffeomorphic to an open ball at the origin in $\mathbb{R}^{d}$ intersected with $s \leq d$ halfspaces $x_{1} \geq 0, \ldots, x_{s} \geq 0$.

Also, the resolution moves the boundaries between the spline domains away from the vertices of $P_{\epsilon}$ :

Proposition 2 The boundaries between polyhedra of the spline subdivision lift to subvarieties (with corners) of $P_{\epsilon}$ which do not contain the vertices of $P_{\epsilon}$.

\subsection{Gelfand-Leray forms and elementary Laplace integrals}

Using the proposition 1 we can localize the integral (4). Indeed, we can represent

$$
B(t)=|P|^{-1} \int_{P} \mathbf{1}(u(x)<t) d \lambda
$$

as

$$
\begin{gathered}
|P|^{-1} \int_{P_{\epsilon}} \mathbf{1}\left(\pi^{*} u(x)<t\right) d \rho \\
=|P|^{-1} \sum_{\mathcal{F}} \int_{P_{\epsilon}} \phi_{\mathcal{F}}(y) \mathbf{1}\left(\pi^{*} u<t\right) d \rho .
\end{gathered}
$$

Here $\rho=\pi^{*} \lambda$ is the pull-back of the Lebesgue measure (possible as $\pi$ is a diffeomorphism onto the interior of $P$ ), and

$$
\sum_{\mathcal{F}} \phi_{\mathcal{F}}=1, \phi_{\mathcal{F}} \geq 0
$$

is the partition of unity such that $\phi_{\mathcal{F}}=0$ outside of the small vicinity of the stratum of $P_{\epsilon}$ corresponding to flag $\mathcal{F}$.

The advantage of the decomposition (7) stems from the following 
Lemma 3 Let $y$ be an interior point of a s-dimensional facet of $P_{\epsilon}$ corresponding to a flag $\mathcal{F}=\left(f_{1} \subset\right.$ $\left.f_{2} \subset \ldots \subset f_{l}\right)$. In particular, one can choose a coordinate system centered at $y$ such that near the origin, the polyhedron $P_{\epsilon}$ is given by $\left\{y_{1} \geq 0, \ldots, y_{l} \geq 0\right\}$. Then

- the density of $\rho$ behaves as

$$
\frac{d \rho}{d \lambda}(y)=U_{\rho}(y) \prod_{1}^{s} y_{i}^{c_{i}-1}
$$

and the lift of the function $u$ behave

$$
\pi^{*} u(y)=U_{u}(y) \prod_{1}^{s} y_{i}^{m_{i}}
$$

Here $c_{i}=c\left(f_{i}\right)$ and $m_{i}=m\left(f_{i}\right)$, and $U_{\rho}, U_{u}$ are nonvanishing functions, $U_{\rho}$ smooth, and $U_{u}$ continuous and smooth in vicinities of the vertices of $P_{\epsilon}$.

Now we could apply directly the results of [1], expressing the asymptotics of the "elementary Laplace integral"

$$
\int_{y \geq 0} U(y) e^{-t \prod_{i} y_{i}^{m_{i}}} y_{1}^{c_{1}-1} \cdot \ldots \cdot y_{s}^{c_{s}-1} d y_{1} \ldots d y_{s},
$$

or, more directly, can analyze the poles of the Mellin transform

$$
\int_{y}\left(\pi^{*} u(y)\right)^{z} y_{1}^{c_{1}-1} \cdot \ldots \cdot y_{s}^{c_{s}-1} d y_{1} \ldots d y_{s}
$$

and apply the results of [10] relating them to the Laplace integrals. One can then see that the leading terms of the asymptotics come from vicinities of the vertices of $P_{\epsilon}$, leading to Theorem 2 .

\section{4 concluding remarks}

- The main results of this note give an algorithm of computing the asymptotics of $M_{n}$, in the polyhedral setup. The method requires, on its face, to enumerate and to analyze all the flags of a polyhedron, the number of which grows superexponentially with the dimension. There are obvious shortcuts, and a more efficient way to find the growth rates of $M_{n}$ might be quite feasible.

- What happens if the cone $K$ is not polyhedral but rather semi-algebraic? A lot of the elements of the proofs survive; but some auxiliary results (especially Lemma 3) would need some rethinking.

\section{References}

[1] Arnold, V. I., Gusein-Zade, S. M., Varchenko, A. N. Singularities of differentiable maps. Vol. II. Monodromy and asymptotics of integrals. Monographs in Mathematics, 83. Birkhuser, Boston, MA, (1988).

[2] Barndorff-Nielsen, O. and Sobel, M. On the distribution of the number of admissible points in a vector random sample. Theory Probab. Appl. 11 249-269. (1966) 
[3] Baryshnikov, Yu. M. Mathematical expectation of the number of variants that are nondominated with respect to a binary relation. Automat. Remote Control 46, no. 6, part 2, 774-779 (1985).

[4] Bentley, J. L., Clarkson, K. L. and Levine, D. B. Fast linear expected-time algorithms for computing maxima and convex hulls. Algorithmica 9 168-183 (1993).

[5] Bentley, J. L., Kung, H. T., Schkolnick, M. and Thompson, C. D. On the average number of maxima in a set of vectors and applications. J. Assoc. Comput. Mach. 25 536-543 (1978).

[6] Buchta, C. On the average number of maxima in a set of vectors. Inform. Process. Lett. 33 63-65 (1989).

[7] Golin, M. J. How many maxima can there be? Comput. Geom. 2 (1993), no. 6, 335-353.

[8] Golin, M. J. Maxima in convex regions. Proceedings of the Fourth Annual ACM-SIAM Symposium on Discrete Algorithms (Austin, TX, 1993), 352-360, ACM, New York, 1993.

[9] Devroye, L. A note on finding convex hulls via maximal vectors. Inform. Process. Lett. 11 53-56 (1980).

[10] Gelfand, I. M., Shilov G.E.Generalized Functions, vol. 1 Academic Press, San Diego (1964). 
